\title{
Stage III Gastric and Omental Gastrointestinal Stromal Tumor AJCC v8
}

National Cancer Institute

\section{Source}

National Cancer Institute. Stage III Gastric and Omental Gastrointestinal Stromal Tumor

A/CC v8. NCl Thesaurus. Code C136774.

Stage III includes: IIIA: (T3, NO, MO, High Mitotic Rate); IIIB: (T4, NO, MO, High Mitotic Rate). T3: Tumor measuring more than $5 \mathrm{~cm}$ but not more than $10 \mathrm{~cm}$. T4: Tumor measuring more than $10 \mathrm{~cm}$ in greatest dimension. NO: No regional lymph node metastasis or unknown lymph node status. M0: No distant metastasis. High Mitotic Rate: Over 5 mitoses per 5 square millimeters, or per 50 HPF. (AJCC 8th ed.) 